\title{
CORRECTIONS TO: SPACE FILLING CURVES OVER FINITE FIELDS
}

\author{
Nicholas M. Katz
}

\section{Introduction}

Ofer Gabber has kindly pointed out to me that the proof of Lemma 5 in my article, "Space filling curves over finite fields," which appeared in Mathematical Research Letters, Volume 6, Number 5-6, pp. 613-624, is wrong. The error occurs in the last paragraph of the proof. The first sentence of that paragraph makes a false statement about Frobenius elements (starting with "so every..."). This false statement is used in the following sentence ("Therefore...") to assert that, for $r \geq r_{0}$, the conditions $\left(\star \star r, \mathcal{D}_{r}, \mathcal{C}_{r}\right)$ and $(\star \star r, \mathcal{E} / W)$ are equivalent. It is indeed trivially true that the first condition implies the second, but the converse need only hold if in addition $r$ is divisible by $N$, the order of the cyclic group $\Gamma / \Gamma_{\text {geom }}$. The effect of correcting this error is that in Lemmas 4,5 , and 6 , and in Corollary 7, what is asserted to hold for $r$ sufficiently large holds only for $r$ sufficiently large and sufficiently divisible. Indeed, Gabber has constructed examples to show that Lemma 6 and Corollary 7 can be false without this extra proviso. In the corrections below, we also modify the statement of Lemma 5 so that its new, weaker conclusion applies in a more general setting.

\section{Corrections and modifications to statements of results}

page 616, assertion 2) of Lemma 4: should read "For all sufficiently large and sufficiently divisible $r, D_{r} / k$ is geometrically connected.".

page 617 , lines $7-8$ of the statement of Lemma 5 (i.e., lines 13-14 on the page): should read " $r \geq r_{0}$, a smooth, geometrically connected $k$-scheme $\mathcal{C}_{r}$ and a $k$-morphism $i_{r}: \mathcal{C}_{r} \rightarrow W$ which is surjective on $k_{r}$-valued points. Form the fibre"

page 617 , line 12 of the statement of Lemma 5 (i.e., line 18 on the page): the label of the map in the diagram should be $i_{r}$ and not $i_{r, W}$.

page 617, last line of the statement of Lemma 5: should read "Then for $\mathrm{r}$ sufficiently large and sufficiently divisible, the fibre product $\mathcal{D}_{r} / k$ is geometrically connected."

page 619, assertion a) of Lemma 6: should read "For r sufficiently large and sufficiently divisible, we have an equality of images of geometric fundamental

Received September 1, 2001. 
groups

(equality inside G)."

$$
\rho_{r}\left(\pi_{1}^{\text {geom }}\left(\mathcal{C}_{r}, c_{r}\right)\right)=\rho\left(\pi_{1}^{\text {geom }}(W, w)\right)
$$

page 619, assertion b) of Lemma 6: should read (in its entirety) "For $\mathrm{r}$ sufficiently large and sufficiently divisible, we have an equality of images of fundamental groups

(equality inside G)."

$$
\rho_{r}\left(\pi_{1}\left(\mathcal{C}_{r}, c_{r}\right)\right)=\rho\left(\pi_{1}(W, w)\right)
$$

page 620 , lines -5 and -2 in the statement of Corollary 7 (i.e. lines -10 and -6 on the page): should read respectively "Then (resp. then) for $r$ sufficiently large and sufficiently divisible, the pullback sheaf $\left(f_{r}\right)^{\star}(\mathcal{F})$ on $\mathcal{C}_{r}$ has the same..."

\section{Corrections to the proof of Lemma 5}

page 618, line 15: should read "We will show that for any $r \geq r_{0}$ which is divisible by $N$ and which is large enough that $(\star \star r, \mathcal{E} / W)$ holds, $\mathcal{D}_{r}$ is"

page 618 , line -2 , through page 619 , line 2: Delete the last paragraph of the proof. Replace it by the following text.

We will show that for any $r \geq r_{0}$ which is divisible by $N$ and for which $(\star \star r, \mathcal{E} / W)$ holds, the condition $\left(\star \star r, \mathcal{D}_{r}, \mathcal{C}_{r}\right)$ holds. We now fix one such $r$.

The subgroup $\pi_{1}\left(W \otimes_{k} k_{r}, w\right)$ of $\pi_{1}(W, w)$ maps, by $\rho$, to the subgroup $\Gamma(0)=\Gamma_{\text {geom }}$, simply because $N$ divides $r$. Because this subgroup contains $\pi_{1}^{\text {geom }}(W, w)$, it maps onto $\Gamma_{\text {geom }}$. This subgroup contains all the Frobenius elements in $\pi_{1}(W, w)$ attached to $k_{r}$-valued points of $W$. By $(\star \star r, \mathcal{E} / W)$, the images of these elements fill $\Gamma_{\text {geom }}$. By the spacefilling property, each of these elements is $\pi_{1}\left(W \otimes_{k} k_{r}, w\right)$-conjugate to the image of a Frobenius element in $\pi_{1}\left(\mathcal{C}_{r} \otimes_{k} k_{r}, c_{r}\right)$ attached to a $k_{r}$-valued point of $\mathcal{C}_{r}$. The group $\pi_{1}\left(\mathcal{C}_{r} \otimes_{k} k_{r}, c_{r}\right)$ maps to $\Gamma_{\text {geom }}$, and the images of its Frobenius elements attached to $k_{r}$-valued

points of $\mathcal{C}_{r}$ meet every conjugacy class in $\Gamma_{\text {geom }}$. So by Jordan's theorem, we conclude that $\pi_{1}\left(\mathcal{C}_{r} \otimes_{k} k_{r}, c_{r}\right)$ maps onto $\Gamma_{\text {geom }}$. Thus every element in $\Gamma_{\text {geom }}$ is the image of a $\pi_{1}\left(\mathcal{C}_{r} \otimes_{k} k_{r}, c_{r}\right)$-conjugate of some Frobenius element attached to a $k_{r}$-valued point of $\mathcal{C}_{r}$ (because the images of these Frobenius elements meet every conjugacy class in $\Gamma_{\text {geom }}$, and $\pi_{1}\left(\mathcal{C}_{r} \otimes_{k} k_{r}, c_{r}\right)$ maps onto $\left.\Gamma_{\text {geom }}.\right)$ But the $\pi_{1}\left(\mathcal{C}_{r} \otimes_{k} k_{r}, c_{r}\right)$-conjugates of Frobenius elements attached to $k_{r}$-valued points of $\mathcal{C}_{r}$ are themselves such Frobenius elements, and thus $\left(\star \star r, \mathcal{D}_{r}, \mathcal{C}_{r}\right)$ holds.

We remark that from the fact that $\pi_{1}\left(\mathcal{C}_{r} \otimes_{k} k_{r}, c_{r}\right)$ maps onto $\Gamma_{\text {geom }}$, it follows that $\pi_{1}\left(\mathcal{C}_{r}, c_{r}\right)$ maps onto $\Gamma$, simply because any element of degree one in the source maps onto a generator of the cyclic quotient $\Gamma / \Gamma_{\text {geom }}$.

\section{Correction to the proof of Lemma 6}

page 619 , line -3: the sentence should end "for $r$ divisible by $N$ and $r \gg 0$." page 619, line-1: the sentence should end "whence a) and b), cf. the corrected proof of Lemma 5."

page 620 , delete lines 1-4. 
page 620, line 10: add the words "and sufficiently divisible" after the phrase "for $r \gg 0$ ".

\section{Correction to the proof of Theorem 8}

page 621, line 7: should read "By Lemma 4, for large $r$ which is sufficiently divisible, this closed subscheme $\mathcal{D}_{r d}$ of $\mathrm{V}$ is a smooth"

\section{Counterexamples}

In this section, we construct, following ideas of Ofer Gabber, counterexamples to the uncorrected versions of Lemma 6 and Corollary 7 . We work over the finite field $k=\mathbb{F}_{q}$. Let $G / k$ be a smooth, geometrically connected, commutative group-scheme of dimension $n \geq 1$, and

$$
\phi: G \rightarrow G
$$

a finite etale homomorphism of $k$-group-schemes of degree $\operatorname{deg}(\phi) \geq 2$ with the following property: the finite etale commutative $k$-group-scheme $\operatorname{Ker}(\phi)$ has no nontrivial $k$-rational points, i.e., $\operatorname{Ker}(\phi)(k)=\{e\}$. Here are three elementary examples of such situations $(G, \phi)$.

(1) Pick $\alpha \neq 1$ in $\mathbb{F}_{q}^{\times}$, take $G=\mathbb{G}_{a}, \phi(x):=x^{q}-\alpha x$. Here $\operatorname{deg}(\phi)=q$.

(2) Pick a prime number $l \geq q+1$, take $G=\mathbb{G}_{m}, \phi(x):=x^{l}$. Here $\operatorname{deg}(\phi)=l$.

(3) Pick a prime number $l \geq 2+q+2 \sqrt{q}$ and an elliptic curve $E / \mathbb{F}_{q}$, take $G=E, \phi(P):=l P$. Here $\operatorname{deg}(\phi)=l^{2}$.

The geometric Frobenius $F_{k}$ in $\operatorname{Gal}(\bar{k} / k)$ acts as a group-automorphism of the finite group $\operatorname{Ker}(\phi)(\bar{k})$. Denote by $N$ the order of this automorphism. By hypothesis, $F_{k}$ has no fixed points in $\operatorname{Ker}(\phi)(\bar{k})-\{e\}$. Therefore, for any integer $r \geq 1$ with $(r, N)=1,\left(F_{k}\right)^{r}$ has no fixed points in $\operatorname{Ker}(\phi)(\bar{k})-\{e\}$. So for any such $r, \operatorname{Ker}(\phi)\left(k_{r}\right)=\{e\}$, i.e., the map

$$
\phi: G \rightarrow G
$$

is injective, and hence bijective, on $k_{r}$-valued points.

In the notations of Lemma 6 and Corollary 7, take $W=G$, with base point $w=e$. For $r \geq 1$ with $(r, N)=1$, take $f_{r}: \mathcal{C}_{r} \rightarrow W$ to be $\phi: G \rightarrow G$; for other $r$, take it to be $i d: G \rightarrow G$. Take $c_{r}=e$ as base point in $\mathcal{C}_{r}=G$, and take as "chemin" from $f_{r}\left(c_{r}\right)=e$ to $e$ the identity. To make counterexamples to Lemma 6 and Corollary 7 , we need only exhibit a finite etale covering

$$
\pi: \mathcal{E} \rightarrow G
$$

which is geometrically nontrivial, but whose pullback by $\phi: G \rightarrow G$ is geometrically trivial. For the monodromy representation of such a covering will violate conclusion a) of Lemma 6 for every $r \geq 1$ with $(r, N)=1$, and the lisse sheaf $\mathcal{F}:=\pi_{\star} \overline{\mathbb{Q}}_{l}$ on $G$ will violate the first conclusion of Lemma 7 for the same $r$. Such a $\pi: \mathcal{E} \rightarrow G$ is given by $\phi: G \rightarrow G$. 